\title{
SPARTAN RANDOM PROCESSES IN TIME SERIES MODELING
}

\author{
M. Žukoviđ* and D. T. Hristopulos \\ Technical University of Crete
}

(Dated: November 13, 2018)

\begin{abstract}
A Spartan random process (SRP) is used to estimate the correlation structure of time series and to predict (extrapolate) the data values. SRP's are motivated from statistical physics, and they can be viewed as Ginzburg-Landau models. The temporal correlations of the SRP are modeled in terms of 'interactions' between the field values. Model parameter inference employs the computationally fast modified method of moments, which is based on matching sample energy moments with the respective stochastic constraints. The parameters thus inferred are then compared with those obtained by means of the maximum likelihood method. The performance of the Spartan predictor (SP) is investigated using real time series of the quarterly S\&P 500 index. SP prediction errors are compared with those of the Kolmogorov-Wiener predictor. Two predictors, one of which explicit, are derived and used for extrapolation. The performance of the predictors is similarly evaluated.
\end{abstract}




\section{INTRODUCTION}

Time series that carry information about temporal autocorrelations in variables such as stock prices, interest rates, etc. have wide applications in finance [1]. Such information allows predictions of time-series, estimating the associated prediction uncertainty, and performing stochastic simulations for reconstructing realizations of the process. For Gaussian time series, the temporal structure is estimated from the data by calculating the autocovariance matrix or the structure function (variogram). A different approach, typically used in statistical physics, focuses on physical interactions embodied in the energy functional.

Statistical physics plays an increasingly important role in economics, helping to understand the behavior of complex economical and financial systems ([2]). However, applications in the prediction and simulation of such systems are explored less. Recently, a class of Gaussian random fields, named Spartan Spatial Random Fields (SSRF) [3] was proposed as a general framework for spatial modelling. Its main advantages lie in parametric frugality, potential for including physical constraints in the probability density function (pdf), and efficient model estimation. Herein we define Spartan Random Processes (SRP) in time, formulate a Spartan predictor (SP), and investigate its potential for time series prediction.

\section{SPARTAN RANDOM PROCESSES}

Let us consider a noise-free detrended time-series $X_{\lambda}(t)$ that represents the fluctuations of an observable with temporal resolution $\lambda$. For statistical inference from a single realization (state), second-order stationarity [4] and ergodicity [5], 6] are often assumed. The latter implies that the 'characteristic' scale of the fluctuations be considerably smaller than the domain size. In statistical physics, the pdf of stationary Gaussian time series can be expressed in terms of an energy functional $H\left[X_{\lambda}(t)\right]$, according to the Gibbs pdf $f_{\mathrm{x}}\left[X_{\lambda}\right]=Z^{-1} \exp \left\{-H\left[X_{\lambda}\right]\right\}$, where $Z$ is a normalizing constant (the partition function).

The SRPs can be defined following the formalism introduced in [3], restricted to one dimensional domains. The fluctuation-gradient-curvature (FGC) SSRF model introduced in [3] embodies Gaussian fluctuations and involves three terms that measure the square of the magnitude, the gradient and the curvature of the fluctuations. On a 1D chain, the FGC form of $H\left[X_{\lambda}\right]$ can generally be written as 


$$
H_{\mathrm{fgc}}\left[X_{\lambda} ; \boldsymbol{\theta}\right]=\frac{1}{2 \eta_{0} \xi} \int d t\left\{\left[X_{\lambda}(t)\right]^{2}+\eta_{1} \xi^{2}\left[\dot{X}_{\lambda}(t)\right]^{2}+\xi^{4}\left[\ddot{X}_{\lambda}(t)\right]^{2}\right\}
$$

where $\dot{X}_{\lambda}(t)$ and $\ddot{X}_{\lambda}(t)$ denote respectively the first and second time derivatives, and $\boldsymbol{\theta}=$ $\left(\eta_{0}, \eta_{1}, \xi, k_{c}\right)$ is a vector of model parameters: $\eta_{0}$ is the scale coefficient, $\eta_{1}$ the autocovariance shape coefficient, $\xi$ is the characteristic length, and $k_{c} \propto \lambda^{-1}$ the cutoff frequency. For discrete time series sampled at $t_{i}=i \alpha, i=1, \ldots, N, \alpha>0$, the energy functional can be expressed in terms of local energies $S_{m}\left(t_{n}\right), m=0,1,2$, as follows:

$$
H_{\mathrm{fgc}}\left[X_{\lambda} ; \boldsymbol{\theta}\right]=\frac{1}{2 \eta_{0} \xi} \sum_{n=1}^{N}\left\{S_{0}\left(t_{n}\right)+\eta_{1} \xi^{2} S_{1}\left(t_{n}\right)+\xi^{4} S_{2}\left(t_{n}\right)\right\},
$$

where $S_{0}\left(t_{n}\right)=X_{\lambda}^{2}\left(t_{n}\right), S_{1}\left(t_{n}\right)=\left[\left(X_{\lambda}\left(t_{n}+\alpha\right)-X_{\lambda}\left(t_{n}\right)\right) / \alpha\right]^{2}$ is the square of the forwarddifference gradient approximation, and $S_{2}\left(t_{n}\right)=\left[X_{\lambda}\left(t_{n}+\alpha\right)+X_{\lambda}\left(t_{n}-\alpha\right)-2 X_{\lambda}\left(t_{n}\right)\right]^{2} / \alpha^{4}$ is the square of the discrete approximation of the Laplacian. The $1 \mathrm{D}$ spectral density is given by the following expression

$$
\tilde{G}_{\mathrm{x}}(k ; \boldsymbol{\theta})=\frac{\tilde{K}_{\lambda}(k) \eta_{0} \xi}{1+\eta_{1}(k \xi)^{2}+(k \xi)^{4}},
$$

where $\tilde{K}_{\lambda}(k)$ is the Fourier transform $(\mathrm{FT})$ of the smoothing kernel that imposes the resolution $\lambda$. The autocovariance function is obtained from the inverse FT, i.e,. by the integral

$$
G_{\mathrm{x}}(t ; \boldsymbol{\theta})=\int_{-\infty}^{\infty} \frac{d k}{2 \pi} \tilde{G}_{\mathrm{x}}(k ; \boldsymbol{\theta}) \exp (\jmath k t)
$$

\section{PARAMETER INFERENCE: THE MODIFIED METHOD OF MOMENTS}

The modified method of moments (MMoM) is based on fitting sample constraints with corresponding stochastic ones. The former are based on the short-range moments $S_{m}\left(t_{n}\right), m=0,1,2$, appearing in Eq. (2). They are evaluated by means of sample averages: $\overline{S_{0}\left(t_{n}\right)}, \overline{S_{1}\left(t_{n}\right)}$, and $\overline{S_{2}\left(t_{n}\right)}$. The respective stochastic constraints can be expressed as follows:

$$
\begin{gathered}
E\left[S_{0}\right]=\mathrm{G}_{\mathrm{x}}(0)=\frac{\eta_{0} \xi}{\pi} \int_{0}^{\mathrm{k}_{\mathrm{c}}} \mathrm{dk} \frac{1}{1+\eta_{1}(\mathrm{k} \xi)^{2}+(\mathrm{k} \xi)^{4}}, \\
E\left[S_{1}\right]=\frac{2}{\alpha^{2}}\left[\mathrm{G}_{\mathrm{x}}(0)-\mathrm{G}_{\mathrm{x}}(\alpha)\right]=\frac{2 \eta_{0} \xi}{\pi \alpha^{2}} \int_{0}^{k_{c}} d k \frac{[1-\cos (k \alpha)]}{1+\eta_{1}(k \xi)^{2}+(k \xi)^{4}},
\end{gathered}
$$




$$
E\left[S_{2}\right]=\frac{2}{\alpha^{4}}\left[3 \mathrm{G}_{\mathrm{x}}(0)+\mathrm{G}_{\mathrm{x}}(2 \alpha)-4 \mathrm{G}_{\mathrm{x}}(\alpha)\right]=\frac{2 \eta_{0} \xi}{\pi \alpha^{4}} \int_{0}^{k_{c}} d k \frac{[3+\cos (2 k \alpha)-4 \cos (k \alpha)]}{1+\eta_{1}(k \xi)^{2}+(k \xi)^{4}} .
$$

The stochastic constrains are related to the SRP model and depend on $\alpha, \eta_{0}, \eta_{1}$, $\xi$, and $k_{c}$. For the uniform sampling step $\alpha$ used herein an infinite $k_{c}$ (unlimited-band), will be considered, allowing the stochastic constraints Eqs. (5) to be be expressed in closed form. The model parameters are estimated by minimizing the following distance metric [3]

$$
\Phi_{s}[X(s)]=\left|1-\sqrt{\overline{\overline{S_{1}}} \frac{E\left[S_{0}\right]}{E\left[S_{1}\right]}}\right|^{2}+\left|1-\sqrt{\frac{\overline{S_{2}}}{\overline{S_{1}}} \frac{E\left[S_{1}\right]}{E\left[S_{2}\right]}}\right|^{2} .
$$

\section{FCG MODEL BASED SPARTAN PREDICTOR}

Let us assume that $T_{m}=\left\{t_{1}, \ldots, t_{K}\right\}$ is a set of sampling times and $X\left(T_{m}\right)=$ $\left\{X\left(t_{1}\right), \ldots, X\left(t_{K}\right)\right\}$ the set of measurements. The spacing of $T_{m}$ is either uniform (full series) or non-uniform (training subset). We assume that $T_{p}=\left\{z_{1}, \ldots, z_{k}\right\}$ is a set of estimation points, disjoint from $T_{m}$, and $\hat{X}\left(T_{p}\right)$ are the estimated values (temporal predictions). $T=T_{m} \cup T_{p}$ is the full measurement - prediction set with cardinality $N=K+k$.

Interpolation: The Spartan predictor $(\mathrm{SP})$ is based on maximizing the conditional probability density $f_{X}\left[X\left(T_{p}\right) \mid X\left(T_{m}\right)\right]$. Considering the relation $f_{X}\left[X\left(T_{p}\right) \mid X\left(T_{m}\right)\right]=$ $f_{X}[X(T)] / f_{X}\left[X\left(T_{m}\right)\right]$, the problem reduces to finding the maximum of $f_{X}[X(T)]$. We ac-

complish this by replacing of $H_{\mathrm{fgc}}$ with the estimator $\hat{H}_{\mathrm{fgc}}[X(T) ; \boldsymbol{\theta}]=\frac{1}{2} X^{\prime}(T) J_{\mathrm{x}}(\boldsymbol{\theta}) X(T)$, and solving the linear system

$$
\partial \hat{H}_{\mathrm{fgc}}[X(T) ; \boldsymbol{\theta}] /\left.\partial X\left(z_{l}\right)\right|_{\hat{X}\left(z_{l}\right)}=0, \quad l=1, \ldots, k .
$$

$\hat{H}_{\mathrm{fgc}}[X(T) ; \boldsymbol{\theta}]$ involves the sampling points as well as the prediction points, and $J_{\mathrm{x}}(\boldsymbol{\theta})$ only depends on the model parameters, not the data. Neglecting interactions between the prediction points, the linear predictor can be expressed explicitly by

$$
\hat{X}\left(T_{p}\right)=-\sum_{L \in V} J_{\mathrm{x}}\left(T_{L}, T_{p}\right) X\left(T_{L}\right) / J_{\mathrm{x}}\left(T_{L}, T_{L}\right),
$$

where $V$ is the interaction neighborhood. Herein, the latter extends up to the secondnearest neighbor. The numerical complexity of SP involved in solving simultaneously $k$ coupled Eqs. (9) is $O\left(k^{3}\right)$. The predictor given by Eq. (10) is explicit. 
Extrapolation: Given the set of measurements $X\left(T_{m}\right)=\left\{X\left(t_{1}\right), \ldots, X\left(t_{N}\right)\right\}$ at times $T_{m}=\left\{t_{1}, \ldots, t_{N}\right\}$, we aim to estimate $k$ future values, $\hat{X}\left(T_{p}\right) p=N+1, \ldots, N+k$. Two approaches are possible: (i) Multipoint (MP) extrapolation, i.e. solving simultaneously the system of equations (9), where $l=N+1, \ldots, N+k$, or (ii) iterative feedforward (IFF) point-like prediction. The latter is based on the short-range memory property $f_{X}\left[X\left(t_{l}\right) \mid X\left(\left\{t_{l-3}, t_{l-2}, t_{l-1}\right\}\right)\right]=f_{X}\left[X\left(t_{l}\right) \mid X\left(\left\{t_{l-2}, t_{l-1}\right\}\right)\right]$, which allows the following explicit predictor, where $\hat{X}\left(t_{i}\right)=X\left(t_{i}\right)$ for $i=N-1, N$ :

$$
\hat{X}\left(t_{l}\right)=-\left[J_{l-2, l} \hat{X}\left(t_{l-2}\right)+J_{l-1, l} \hat{X}\left(t_{l-1}\right)\right] / J_{l, l} \quad l=N+1, \ldots, N+k .
$$

\section{DESCRIPTION OF DATA AND METHODS OF ANALYSIS}

The time series used in this study consists of 388 quarterly ( $\alpha=1 / 4$ year) S\&P 500 index data, recorded in 1900-1996 [7]. The Spartan parameters are estimated by means of the MMoM and the maximum likelihood estimation (MLE) method [8], using a training sets of 132 points. These are randomly selected from the 388 points to obtain 100 different configurations. The optimization uses the Nelder-Mead simplex search algorithm [9] and is terminated when both the model parameters and the cost function change between consecutive steps less than $\epsilon=10^{-6}$. The cost function is given by the negative log-likelihood -NLL- function in the case of MLE, and the distance metric -DM-, given by Eq. (8), in the MMoM case. Initial guesses for the Spartan model parameters are $\xi_{i}^{(0)}=\alpha$ and $\eta_{1}^{(0)}=1$.

To evaluate the prediction performance, for each realization of the training set, the remaining 256 points (validation set) are predicted by both SP (MP and IFF) and KolmogorovWiener predictor (KWP) [10]. In both cases, the Spartan covariance model is used. In KWP, the search neighborhood includes the entire series. The following statistics are evaluated, where $X_{i}$ is the real value, $\hat{X}_{i}$ is the estimate, and $M$ is the number of validation points: (i) mean absolute error (MAE): $\sum_{i=1}^{M}\left|X_{i}-\hat{X}_{i}\right| / M$ (ii) mean relative error (MRE): $\sum_{i=1}^{M}\left(X_{i}-\hat{X}_{i}\right) / X_{i}$ (iii) mean absolute relative error (MARE): $\sum_{i=1}^{M}\left|\left(X_{i}-\hat{X}_{i}\right) / M X_{i}\right|$ (iv)

root mean square error (RMSE): $\sqrt{\sum_{i=1}^{M}\left|X_{i}-\hat{X}_{i}\right|^{2} / M}$, and (v) the linear correlation coefficient $(\mathrm{R})$. The computations are performed in the Matlab® environment on a desktop computer with a Pentium $4 \mathrm{CPU}$ at $3 \mathrm{GHz}$ and $1 \mathrm{~GB}$ of RAM. 


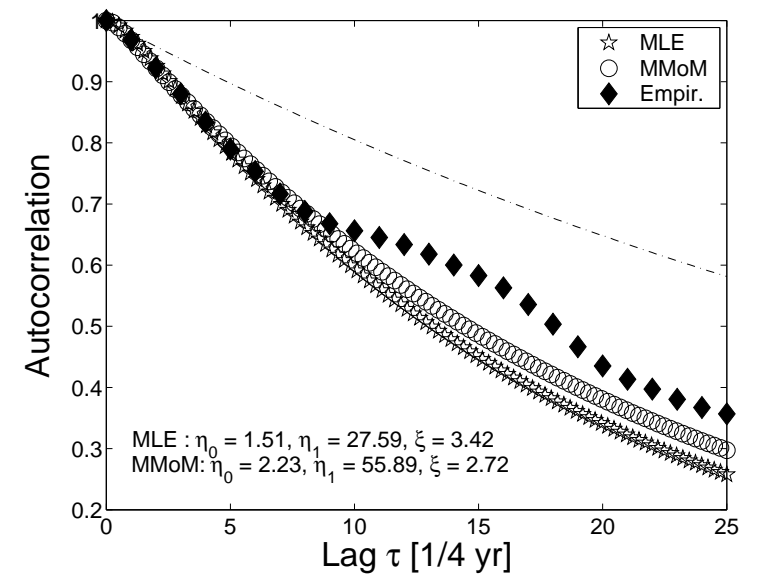

FIG. 1: Empirical correlation function $(\diamond)$ and optimal Spartan model obtained from complete data by means of $\operatorname{MLE}(\star)$ and MMoM (o); for dash-dot line, see text.

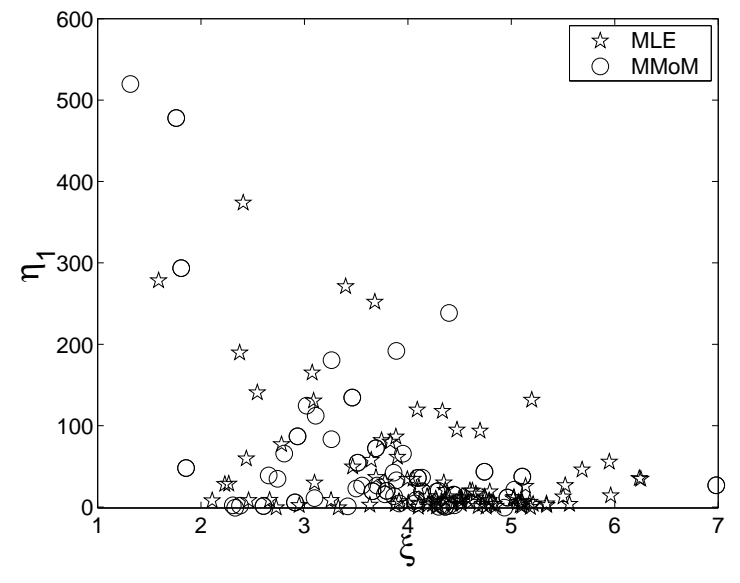

FIG. 2: Estimates of $\xi$ and $\eta_{1}$, obtained from 100 training sets of 132 points by MMoM (०) and MLE ( $\star$. Note: in the case of MMoM some estimates lie outside the axes' range.

\section{RESULTS}

Estimation of Correlations: The empirical correlation function is compared with those obtained with the MMoM and the MLE estimators in Fig. 1, They all match very well near the origin, which is crucial for interpolation. The value of the MMoM distance metric function is $\Phi=8.2 \times 10^{-20}$, indicating excellent match of the sample and stochastic constraints. The optimization CPU time of MMoM (0.078 s) is 228 times faster than the MLE one $(17.08 \mathrm{~s})$. Unlike the MLE CPU time that increases nonlinearly with the data, the MMoM CPU time is insensitive to the domain size [3]. The difference between the two methods in computational time is expected to increase dramatically with the sample size.

As shown in Fig. 2, the distribution of parameter estimates, obtained from different training sets, is scattered and skewed for both MMoM and MLE cases. Some MMoM estimates receive extreme values, e.g. $\eta_{0}>10^{2}, \eta_{1}>10^{3}$, and $\xi<10^{0}$ (not shown in Fig. 2), producing an almost linear decrease of the correlations (e.g., dash-dot line in Fig. 1 corresponds to $\xi=0.065, \eta_{1}=5 \times 10^{5}$ ). Such cases are marked by relatively high $\Phi$ values, indicating poor matching of the sample and stochastic moments, likely due to non-ergodic conditions. Indeed, the integral scale [11] is quite large $I\left(\eta_{1}=55.89, \xi=2.72\right)=41.4$, compared to the time series' length. 
TABLE I: Interpolation errors based on 100 training sets of 132 points.

\begin{tabular}{lccccc}
\hline \hline & MAE & MARE & MRE & RMSE & R \\
\hline MMoM & 0.0717 & 0.0763 & -0.0168 & 0.1174 & 0.9519 \\
MLE & 0.0731 & 0.0771 & -0.0140 & 0.1202 & 0.9493 \\
\hline \hline
\end{tabular}

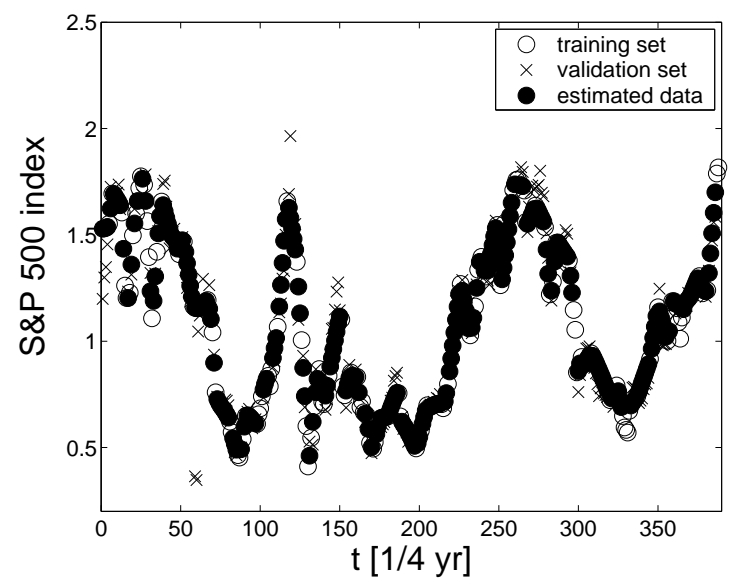

FIG. 3: Time series reconstruction by interpolation: training $(\circ)$, validation $(\times)$, estimates $(\bullet)$.

Interpolation: In the absence of long-range correlations, the short distances are most relevant for interpolation. This is evidenced from Table I, which compares the interpolation errors of the MLE- and MMoM-based estimates: the non-ergodic effect does not introduce significant deviations in the MMoM-based estimates. We evaluate the performance of the interpolation methods by comparing the observations at the validation points with the predictions, using the statistical measures of performance defined above. The model parameters estimates are based on the MMoM. In Table II, the SP prediction errors are compared with those of KWP. The comparison distinguishes between areas with different densities of training data. Nine categories are defined according to the number of training-point neighbors in the vicinity of the validation point. The category $(i, j)$ implies points from the validation set with $i$ nearest and $j$ next-nearest neighbors that belong in the training set. Naturally, the categories with more data in their interaction neighborhood display smaller errors than those with fewer data. There are no significant differences between the SP and KWP results. The linear correlation coefficient values (0.952 for SP and 0.953 for KWP) indicate strong correlation between the predictions and the actual data. An example of a reconstructed time series from 132 points, obtained by means of SP is shown in Fig. 3. 
TABLE II: Interpolation errors of SP and KWP predictions based on 100 training configurations.

\begin{tabular}{|c|c|c|c|c|c|c|c|c|c|c|}
\hline & $(2,2)$ & $(1,2)$ & $(0,2)$ & $(0,1)$ & $(0,0)$ & $(1,1)$ & $(1,0)$ & $(2,0)$ & $(2,1)$ & Total \\
\hline \multicolumn{11}{|c|}{ Mean Absolute Error } \\
\hline SP & 0.041 & 0.050 & 0.063 & 0.085 & 0.126 & 0.051 & 0.053 & 0.036 & 0.034 & 0.0718 \\
\hline KWP & 0.042 & 0.051 & 0.063 & 0.085 & 0.123 & 0.052 & 0.053 & 0.036 & 0.034 & 0.0717 \\
\hline \multicolumn{11}{|c|}{ Mean Absolute Relative Error } \\
\hline SP & 0.038 & 0.052 & 0.067 & 0.097 & 0.129 & 0.054 & 0.059 & 0.039 & 0.033 & 0.0756 \\
\hline KWP & 0.040 & 0.053 & 0.067 & 0.097 & 0.126 & 0.055 & 0.059 & 0.039 & 0.033 & 0.0761 \\
\hline \multicolumn{11}{|c|}{ Mean Relative Error } \\
\hline SP & -0.001 & -0.005 & -0.010 & -0.034 & -0.032 & -0.010 & -0.016 & -0.003 & -0.001 & -0.0168 \\
\hline KWP & -0.001 & -0.004 & -0.009 & -0.034 & -0.032 & -0.010 & -0.015 & -0.002 & 0.000 & -0.0160 \\
\hline \multicolumn{11}{|c|}{ Root Mean Square Error } \\
\hline SP & 0.051 & 0.073 & 0.089 & 0.130 & 0.174 & 0.082 & 0.084 & 0.052 & 0.049 & 0.1174 \\
\hline KWP & 0.053 & 0.075 & 0.089 & 0.130 & 0.165 & 0.082 & 0.084 & 0.053 & 0.050 & 0.1162 \\
\hline
\end{tabular}

Extrapolation: In Table III we compare SP errors, obtained by the SP-MP and SP-IFF methods with those obtained using a second-order autoregressive (AR) model. We randomly select from the $N=388$ data a point $i(2<i<N-k)$ and predict the following $k=3$ values. The errors obtained by the two SP methods are almost identical and comparable (slightly smaller) with those obtained from the AR model. At 1-st, 2-nd and 3-rd lag, the predicted future values have average relative errors on the order of $5 \%, 10 \%$, and $15 \%$, respectively.

\section{SUMMARY}

We present a framework for the analysis of time series based on 'pseudo-energy' functionals that capture the temporal heterogeneity of the observed process. Estimates of the process at unmeasured points (predictions) are based on the mode of the joint pdf. The Spartan prediction method is tested on financial data (S\&P 500 index time series), by both interpolation and extrapolation. The SP yields results well comparable to those obtained by the standard Kolmogorov-Wiener predictor at generally lower computational cost. 
TABLE III: Extrapolation error statistics of SP (MP and IFF) and AR model based on 100 randomly selected triplets $i+1, i+2, i+3(2<i<N-3)$.

\begin{tabular}{lccccccccc}
\hline \hline Method & \multicolumn{3}{c}{ SP-MP } & \multicolumn{3}{c}{ SP-IFF } & \multicolumn{3}{c}{ AR } \\
\hline Lag $\tau$ & 1 & 2 & 3 & 1 & 2 & 3 & 1 & 2 & 3 \\
\hline MAE & 0.0549 & 0.0937 & 0.1390 & 0.0548 & 0.0937 & 0.1390 & 0.0583 & 0.1068 & 0.1549 \\
MARE & 0.0495 & 0.0833 & 0.1383 & 0.0495 & 0.0832 & 0.1384 & 0.0533 & 0.0996 & 0.1633 \\
MRE & 0.0047 & 0.0074 & -0.0156 & 0.0048 & 0.0075 & -0.0155 & -0.0096 & -0.0211 & -0.0583 \\
RMSE & 0.0696 & 0.1164 & 0.1857 & 0.0695 & 0.1164 & 0.1858 & 0.0827 & 0.1396 & 0.2110 \\
\hline \hline
\end{tabular}

We also present an efficient parameter inference technique - modified method of moments (MMoM), which is based on fitting of sample and corresponding stochastic short-range constraints. The main advantage of MMoM is low computational complexity (high speed) and independence on the domain size, which makes it suitable for large data sets, difficult to manage by other techniques. Alternatively, the computational ease gives the method potential include time-dependent parameters that are continuously estimated, in a "moving window" approach, to account for potential non-stationarity. Since the Gaussian assumption is often unjustified, we currently focus on formulating a Spartan model capable of representing directly non-Gaussian data, without the need for a normalizing transformation.

This work is partially supported by the Marie Curie Transfer of Knowledge Program, Project SPATSTAT 014135.

* mzukovic@mred.tuc.gr

$\dagger$ dionisi@mred.tuc.gr

[1] N.H. Chan, Time Series: Applications to Finance, Wiley, New York, 2002.

[2] R.N. Mantegna, H.E. Stanley, An Introduction to Econophysics: Correlations and Complexity in Finance, Cambridge University Press, Cambridge, 1999; J.P. Bouchaud, M. Potters, Theory of Financial Risk, Cambridge University Press, Cambridge, 2000; R.N. Mantegna, (Ed.), Proceedings of the International Workshop on Econophysics and Statistical Finance, Physica A (special issue) 269 (1999); J.P. Bouchaud, P. Alstrom, K.B. Lauritsen, (Eds.), Application 
of Physics in Financial Analysis, Int. J. Theor. Appl. Finance (special issue) 3 (2000).

[3] D.T. Hristopulos, Spartan Gibbs random field models for geostatistical applications, SIAM Journal in Scientific Computation 24 (2003) 2125-2162.

[4] M. Yaglom, Correlation Theory of Stationary and Related Random Functions I, Springer, New York, 1987.

[5] R.J. Adler, The Geometry of Random Fields, Wiley, New York, 1981.

[6] C. Lantuejoul, Geostatistical Simulation: Models and Algorithms, Springer, Berlin, 2002.

[7] S.G. Makridakis, S.C. Wheelwright, R.J Hyndman, Forecasting: Methods and Applications, Wiley, New York, 1998.

[8] R.A. Fisher, On an absolute criterion for fitting frequency curves: Messeng. Math. 41 (1912) 155-160.

[9] W.H. Press, S.A. Teukolsky, W.T. Vettering, B.P. Flannery, Numerical Recipes in Fortran, Vol. 1, Cambridge University Press, New York, 1992.

[10] N. Cressie, Statistics for spatial data, Wiley, New York, 1993.

[11] D.T. Hristopulos, S.N. Elogne, Analytic Properties and Covariance Functions of a New Class of Generalized Gibbs Random Fields, IEEE Trans. Inform. Theory, arXiv:cs/0605073v1. 